\title{
Dynamic Studies of Ototoxicity in Mature Avian Auditory Epithelium
}

\author{
KEIKO HIROSE, ${ }^{a}$ LESNICK E. WESTRUM, ${ }^{b}$ JENNIFER S. STONE, ${ }^{a}$ \\ LANCE ZIRPEL, ${ }^{c}$ AND EDWIN W RUBEL ${ }^{a, d}$ \\ ${ }^{a}$ Virginia Merrill Bloedel Hearing Research Center, Department of Otolaryngology-Head \\ and Neck Surgery, University of Washington, Box, 357923, Seattle, \\ Washington 98195, USA \\ ${ }^{b}$ Department of Neurological Surgery, University of Washington, Box 356470, Seattle, \\ Washington 98195, USA \\ ${ }^{c}$ Department of Neurobiology and Anatomy, University of Utah School of Medicine, 50 \\ North Medical Drive, Salt Lake City, Utah 84132, USA
}

\begin{abstract}
Hearing loss induced by ototoxicity is a worldwide problem despite the development of newer antibiotics and chemotherapy agents. The cellular mechanisms responsible for aminoglycoside-induced hearing loss are still poorly understood. We have developed two different methods of studying the dynamic cellular and subcellular changes in the chick auditory sensory epithelium that occur during hair cell death. The first study was performed in mature chicks after a single, high dose injection of gentamicin, which results in the rapid loss of all hair cells in the basal third of the cochlea. Chicks were sacrificed at discrete time points after drug treatment, and transmission electron microscopy was performed to study the ultrastructural changes in basal hair cells during the course of cell death. We noted various changes in the cell morphology including accumulation of cytoplasmic inclusion bodies, dispersion of the cytoplasmic polyribosomes, mitochondrial swelling, and cellular extrusion by $24 \mathrm{~h}$ after injection. The next two studies were performed using tissue cultures from mature avian auditory sensory epithelium. Cultured cells were labeled using vital fluorescent markers, and levels of intracellular calcium and reactive oxygen species within hair cells were studied following aminoglycoside exposure. We identified a dose-dependent increase in the levels of intracellular calcium, which was blocked by an inhibitor of voltage-gated calcium channels. We also found that levels of reactive oxygen species in hair cells greatly increased after exposure to gentamicin, and this response was blocked by two different antioxidants. These studies serve to identify key cellular and molecular changes in hair cells in response to ototoxic drugs. Further study of these processes may lead to a better understanding of how ototoxicity is induced and to potential preventative interventions.
\end{abstract}

${ }^{d}$ To whom correspondence may be addressed. Phone: (206) 543-8360; fax: (206) 616-1828; e-mail: rubel@u.washington.edu 


\section{INTRODUCTION}

Despite the advent of modern antibiotics and newer cancer chemotherapy agents, ototoxicity remains an important cause of hearing loss. The adverse effects of aminoglycoside antibiotics on hearing and balance are well established, but the cellular mechanisms of ototoxicity remain less clear. Animal studies have been extensively pursued in hopes of elucidating some of the important mechanisms of cell damage in the inner ear due to drug damage. Our laboratory has used the avian animal model to examine some of the dynamic cellular and subcellular changes that occur during early exposure to aminoglycoside antibiotics.

The chick basilar papilla has been the focus of numerous studies that we have conducted in studying both hair cell degeneration and hair cell regeneration. While developing methods to study the process of regeneration in the avian cochlea, members of our laboratory witnessed the events that led to hair cell degeneration. Two different techniques that were developed primarily to study the process of regeneration were adapted in order to examine the process of hair cell death. These two techniques and the observations that were made will be described.

\section{OVERVIEW OF THE AVIAN BASILAR PAPILLA}

The anatomy of the chick basilar papilla is structurally quite different from that of the mammalian cochlea, although its function is very similar (FIG. 1). The basilar papilla is tonotopically organized; the basal end encodes high frequencies, as does the basal turn of the mammalian cochlea, while the apical papilla encodes low frequencies. As in mammals, the basal region is preferentially susceptible to aminoglycoside ototoxicity. Avian hair cells, like their mammalian counterparts, receive both efferent and afferent innervation. The tall hair cells, located on the neural edge of the basilar papilla are considered analogous to the inner hair cells of the mammalian cochlea, and the short hair cells, located on the abneural edge, are thought to be analogous to the outer hair cells. Rather than the three rows of outer hair cells and the single row of inner hair cells seen in mammals, the avian basilar papilla, has a uniform pattern of hair cells with a gradual transition from tall to short hair cells.

One of the critical differences between the avian and the mammalian cochlea is the ability of the avian hair cell to regenerate after an ototoxic insult. However, the process of hair cell death in the chick does appear to be similar to that in mammals, and therefore, examination of avian species will undoubtedly provide some insights into the processes of hair cell death in mammals, including hair cell death in humans.

\section{SINGLE INJECTION PROTOCOL}

A protocol involving a single, high-dose injection of gentamicin in post-hatch chicks was developed in our laboratory in order to provide a model system with predictable timing and localization of regions of hair cell damage and regeneration along the chick basilar papilla. ${ }^{1-3}$ Using a single, subcutaneous dose of gentamicin at $200-400 \mathrm{mg} / \mathrm{kg}$, total loss of hair cells in the basal one-third to one half of the chick 


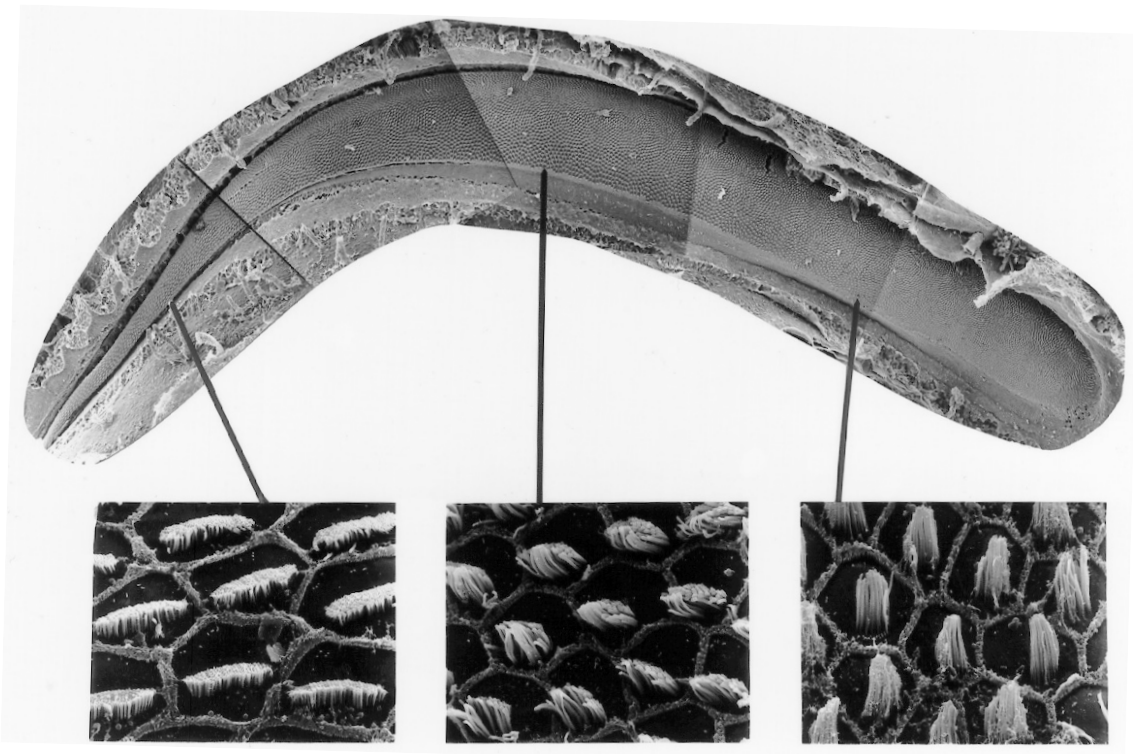

FIGURE 1. Control basilar papilla, low-power scanning electron micrograph of a chick basilar papilla. Insets show higher magnification views of hair cells in the basal (left panel), mid (mid panel), and apical (right panel) regions.

cochlea is observed by 2 days (FIG. 2). ${ }^{4}$ This protocol creates a reliable lesion that occurs early after drug exposure and can be used to perform a more detailed examination of the earliest structural and metabolic changes during hair cell death due to aminoglycoside toxicity.

\section{ULTRASTRUCTURAL CHANGES WITHIN 24 HOURS OF GENTAMICIN TREATMENT}

The primary goal of this study was to document precisely the earliest changes in the nuclei, cellular contacts, and organelles of hair cells during the course of cell death after aminoglycoside exposure. Chicks at post-hatch day 3 received a subcutaneous injection of $300 \mathrm{mg} / \mathrm{kg}$ gentamicin and were sacrificed at very closely spaced intervals: $15,18,21$, and $24 \mathrm{~h}$ postinjection. Basilar papillae were fixed in aldehyde, postfixed in buffered osmium tetroxide solution, dehydrated, and embedded in epoxy resin for conventional transmission electron microscopy. A region of the basilar papilla at $200-400 \mu \mathrm{m}$ from the basal tip was selected. In this region, all hair cells are lost by two days following the injection. Ultrathin sections were taken, and sections were systematically examined. These specimens were compared to sections of control basilar papillae in chicks of the same age. 


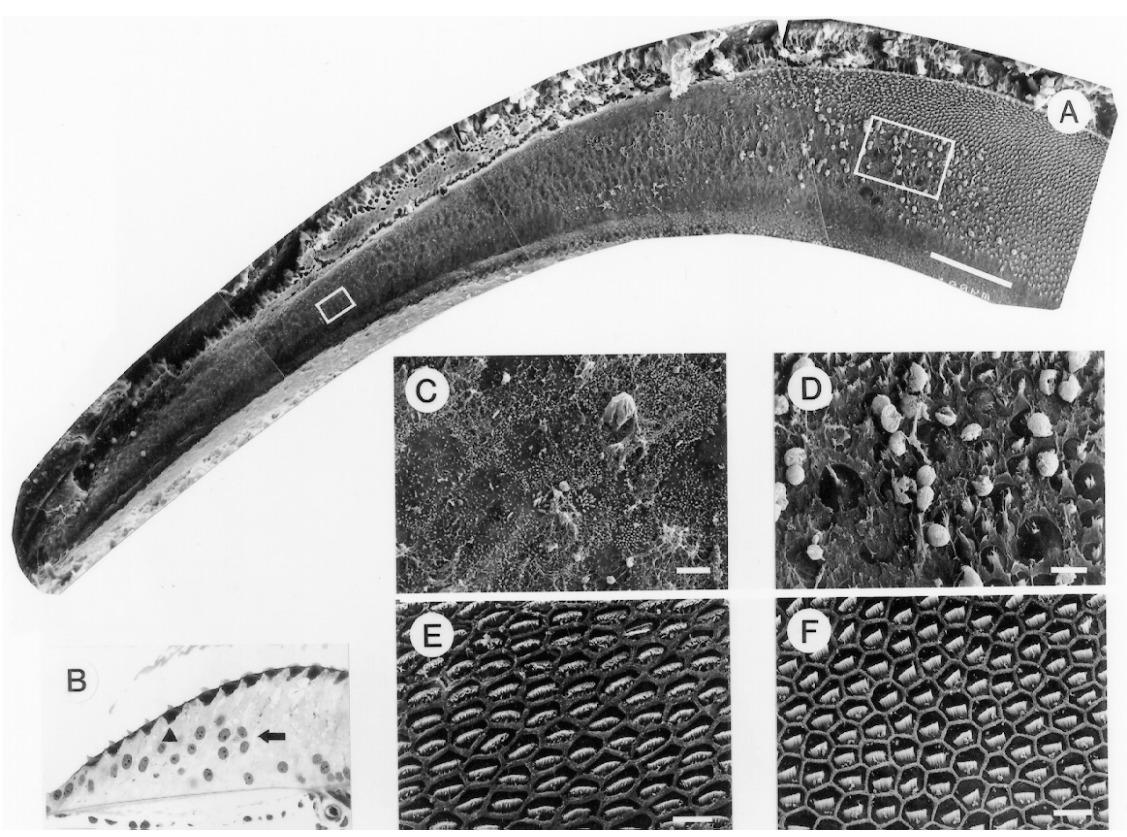

FIGURE 2. (A) Damaged basilar papilla. Scanning electron micrograph of the basal end of a chick basilar papilla, $24 \mathrm{~h}$ after gentamicin injection. Nearly all the hair cells in the basal one third of the organ have been extruded. Scale bar: $100 \mu \mathrm{m}$. (B) Light microscopic view of the basilar papilla cut in cross section. Arrow: Supporting cell; arrowhead: hair cell. Scale bar: $20 \mu \mathrm{m}$. (C) High magnification view of basal region of basilar papilla, damaged. (D) High magnification view of midregion of basilar papilla, damaged. Scale bar: $2 \mu \mathrm{m}$. (E) High magnification view of basal region of basilar papilla, control. Scale bar: $10 \mu \mathrm{m}$. (F) High magnification view of midregion of basilar papilla control. Scale bar: $10 \mu \mathrm{m}$. (From Bhave et al., 1998. ${ }^{4}$ Reprinted with permission.)

Figure 3A shows a low magnification cross section of the basilar papilla from a control animal from the superior to the inferior edge, at $400 \mu \mathrm{m}$ from the basal tip. In this micrograph, the tall-to-short hair cell gradient is well preserved. The inferiorly located shorter hair cells have an angulated, triangular appearance. The distribution of the hair cells among the supporting cells is very regular. A higher magnification view of normal hair cells (FIG. 3B ) demonstrates the typical cytoplasmic features. These include numerous mitochondria, abundant polyribosomes throughout the cytoplasm, and very few cytoplasmic vacuoles. The nuclei have one or two nucleoli, and the nuclear chromatin pattern is dispersed with a slight accumulation around the nuclear envelope. The cell-to-cell contacts with the neighboring supporting cells are very easily identified. Occasional inclusion bodies are seen but are often absent. Afferent and efferent neural apposition are also seen. 

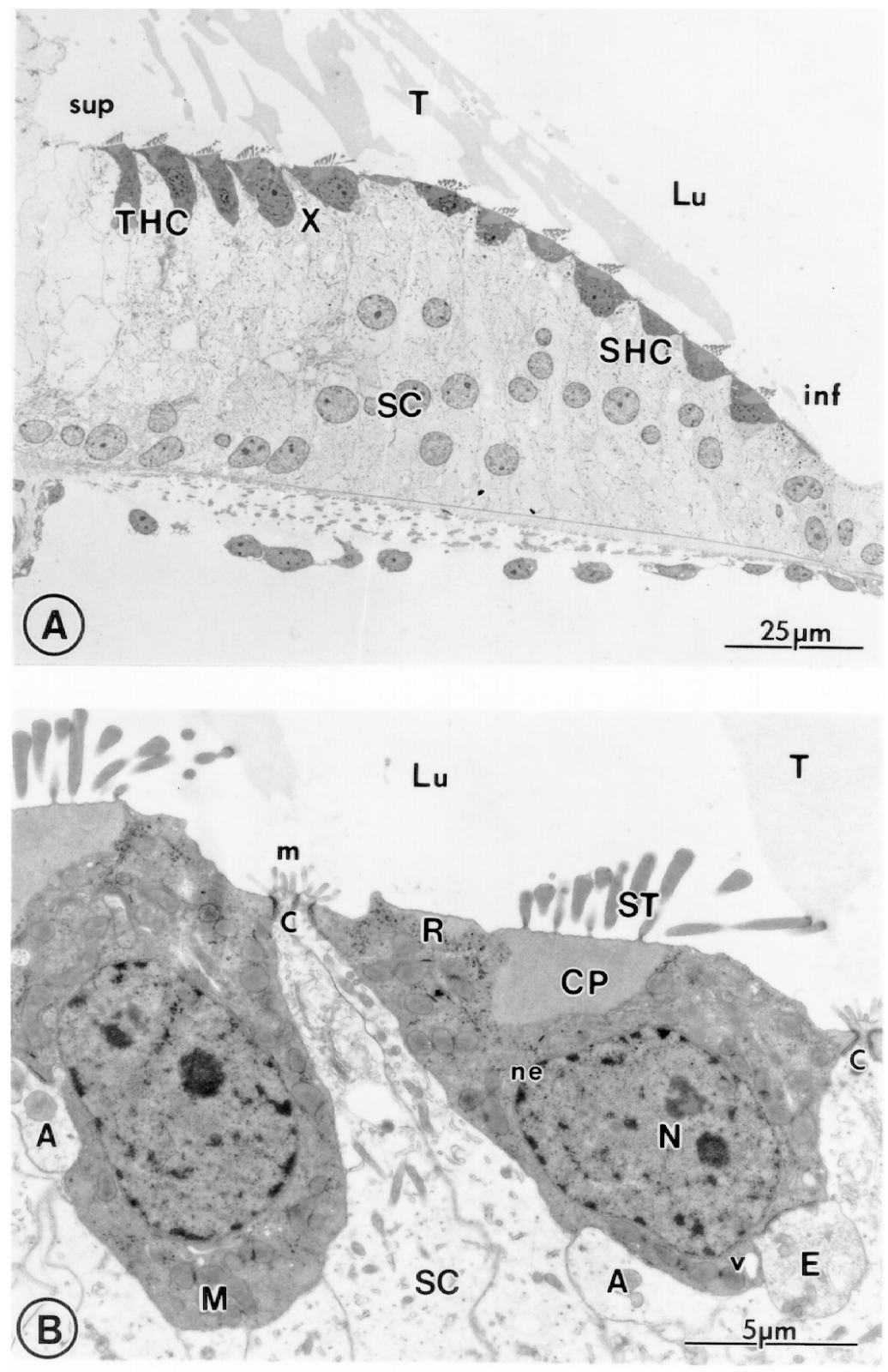

FIGURE 3. (A) Transmission electron micrograph of a normal basilar papilla from a chick post-hatch day 4 at 400 microns from the basal tip. Tall hair cells (THC) occur on the superior (sup) region and short hair cells (SHC) on the inferior (inf) region. Tectorial membrane (T) within the lumen (Lu) abutts the stereocilia. Support(Caption continues on p. 394.) 
By $15 \mathrm{~h}$ after drug exposure, the very first changes in the hair cell ultrastructure were observed (FIG. 4A), although some variation and overlap occur between the early survival times. The majority of the hair cells in these animals appear to be normal at low magnification. However, after drug exposure, a few hair cells, such as the one in the far upper left (see arrow), have already been extruded (FIG. 4B). The drugtreated hair cells are notably more plump and rounded as compared to the angular features of the untreated short hair cells (compare FIG. 4A to FIG. 3A). The stereocilia in dying mammalian hair cells have been described in the past as fused, ${ }^{5,6}$ but in our tissue, the stereocilia are quite normal at this survival time (FIGS. 4A and 4B). Several features are apparent at higher magnification (FIG. 4B). The nuclei are intact and not particularly swollen, but they have irregular clumping of chromatin. The mitochondria are moderately dilated as described in the past. ${ }^{7}$ Various types of inclusion bodies are observed in the cytoplasm of these drug-treated hair cells that are best seen at a higher magnification (FIG. 4B). These inclusions bodies include lysosomes and dark whorled "myelin bodies" that have been described in dying mammalian hair cells. ${ }^{7-9}$ Many of the afferent dendrites in contact with these dying hair cells are very swollen and disrupted, but the efferent endings appear unchanged. The supporting cells and the contacts between the supporting cells and hair cells also appear undisturbed.

Some of the more subtle subcellular changes seen $15 \mathrm{~h}$ after gentamicin are more easily identified at even higher magnification (FIG. 5). Again, the mitochondria are notably dilated. Also, the polyribosomes that are so prominent and regularly distributed in the normal hair cell (FIG. 5A) are completely dispersed within the cells cytoplasm by $15 \mathrm{~h}$ after drug treatment (FIG. 5B). Numerous lysosomes have developed in the cytoplasm. Each of these changes is more pronounced and occur frequently in the subjects by $18-21 \mathrm{~h}$ after injection.

By $24 \mathrm{~h}$ after drug injection, we observed even more markedly abnormal changes in the basal third of the basilar papilla. Several hair cells have formed large cytoplasmic blebs, containing organelles, that project into the lumen (FIG. 6A). Numerous altered hair cells with identifiable stereocilia are fully extruded from the sensory epithelium and trapped under the tectorial membrane. Although the extruded hair cells are swollen and rounded and the nucleus is dilated, the nuclear envelope is identifiable and the chromatin is homogeneously dispersed (FIG. 6B). The hair cell plasma membrane is intact in most cases despite the cytoplasmic swelling. The stereocilia

(FIG. 3, continued.) ing cells (SC) surround the hair cells. The region marked with an $\mathrm{X}$ is enlarged in Figure 3B. (B) Higher magnification of the control basilar papilla, normal structures. Stereocilia (ST) emerge from the cuticular plate (CP) and contact the tectorial membrane (T). The nuclei $(\mathrm{N})$ have 1-2 nucleoli, and chromatin is aggregated on the nuclear envelope (ne). Ribosomes (R), mitochondria (M), and occasional vacuoles (v) are seen, as are afferent (A) and efferent (E) neural contacts. Supporting cells (SC) surround the hair cells and have lumenal microvilli (m) and intercellular contacts (c) with the hair cells. 

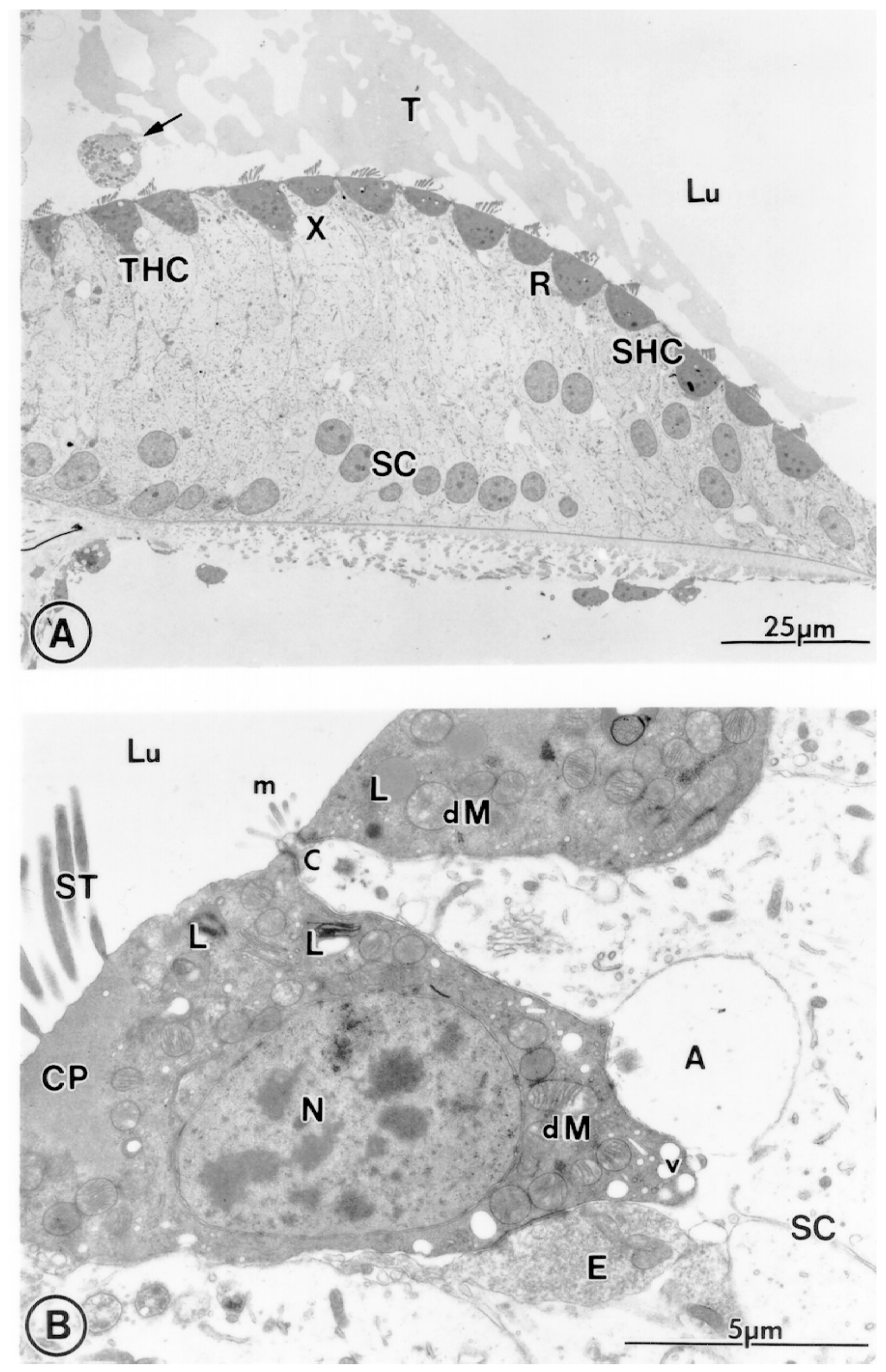

FIGURE 4. (A) Basilar papilla at $15 \mathrm{~h}$ after a single injection of gentamicin in a chick on post-hatch day 3 . One degenerated and extruded hair cell is seen superiorly (arrow), and the short hair cells (SHC) appear more rounded (R) than in normals (compare with FIGURE 3A). Otherwise the papilla appears relatively intact. The area marked with an $\mathrm{X}$ is enlarged below. (B) Higher magnification of hair cells, $15 \mathrm{~h}$

(Caption continues on p. 396.) 
bundle is not fused, and the cuticular plate is present. The contacts between hair cells and supporting cells are preserved in the process of extrusion, which suggests that when these contacts are lost, the loss of the cell junctions is one of the late events during hair cell death. The rounded, extruded hair cell has swollen mitochondria, but some normal-appearing mitochondria as well. Lysosomes and inclusions are seen throughout, but aggregates of ribosomes are lacking and polyribosomes are rare. The cytoplasm has a very lucent appearance, and cytoplasmic vacuolization is prevalent.

By $24 \mathrm{~h}$ after drug injection, there are regions of near total hair cell loss, and in these areas, the reticular lamina is composed entirely of supporting cells (FIGs. 7A and 7B). The supporting cells have normal-appearing intercellular contacts. Occasional inclusion bodies or phagosomes are seen within supporting cells. The supporting cells that replace the extruded hair cells show expansion of the lumenal surface. The microvilli are more separated and sparse on these supporting cells. This bare epithelium is observed along the majority of the basal third of the basilar papilla as early as $24 \mathrm{~h}$ after drug treatment until regeneration begins at $72 \mathrm{~h} .{ }^{10,11}$

A salient feature of gentamicin ototoxicity in chicks is the active extrusion of auditory hair cells from the sensory epithelium. This process of hair cell extrusion has not been consistently described in studies of ototoxicity. However, variations of these events in vestibular explants have been reported, ${ }^{6,12,13}$ and in mammalian organ of Corti as well. . $8,8,14,15$ Initially, this process of hair cell blebbing and extrusion may seem to be unique to the avian inner ear, but in fact, it may be a common process, not only in aminoglycoside ototoxicity, but in development and in other types of hair cell degeneration, such as normal aging.

Apical blebs, early forms of hair cell extrusion, have been described in the literature as early as 1962 . In his paper on vestibular hair cells after streptomycin treatment in cats, Hawkins describes "protoplasmic protrusions" that contain cytoplasmic organelles that are thought to be a normal secretory process of the hair cell, but is much more apparent in the degenerating hair cell. ${ }^{5}$ Sjoback and Wersall indicate that gentamicin treatment in lizards reveals similar "protrusions from the cellular surface into the endolymphatic space" in hair cells of the basilar papilla. ${ }^{7}$ A more recent study, comparing the effects of various ototoxic agents on cultured mouse organ of Corti, demonstrates the presence of apical surface blisters that are again described as "simple protruberances of the cytoplasm ... [which] contain filamentous material, endoplasmic reticulum, vesicles, ribosomes and glycogen."14 These apical surface blisters were described in amikacin and streptomycin toxicity and not routinely with gentamicin in this study. The expelled chick hair cells bear a distinct resemblance to the expelled vestibular hair cells in the jerker mutant mouse. ${ }^{12}$ While the cause of

(FIG. 4, continued.) postinjection. Hair cells have relatively normal stereocilia (ST), cuticular plate (CP) and usual contacts (c) with supporting cells (SC), but clear changes occur in the cells themselves. Nuclear chromatin is dispersed, some mitochondria are dilated $(\mathrm{dM})$, vacuolization is increased $(\mathrm{v})$, and more inclusion bodies or lysosomes (L) can be seen. The afferent dendrite (A) is swollen, but the efferent (E) axon is unaltered. 

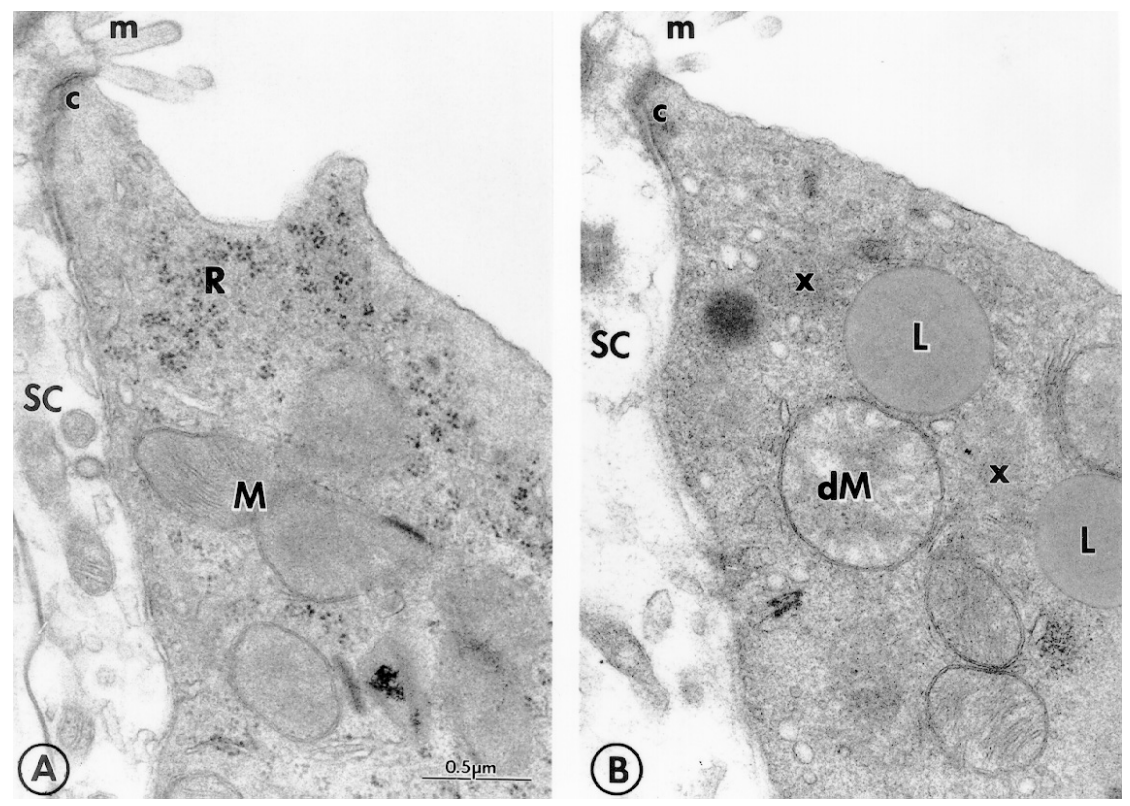

FIGURE 5. (A) High magnification, control hair cell. A portion of a control hair cell (from FIGURE 3B) shows normal-appearing mitochondria (M), ribosomes (R, rosettes and polyribosomes) and contacts (c) with adjacent supporting cells (SC). Microvilli (m) are seen on the lumenal surface of the supporting cells. (B) High magnification, hair cell $15 \mathrm{~h}$ postinjection, the earliest ultrastructural changes. There are dissolution of the ribosomes (x), swelling of the mitochondria $(\mathrm{dM})$, and appearance of more lysosomal inclusion bodies (L). Normal junctions (c) with the adjacent supporting cells (SC) persist.

hair cell degeneration in this mouse is genetic and not toxic, hair cell protrusion from the apical surface and eventual expulsion from the epithelium is remarkably similar to the changes that we see in the chick.

Inclusion bodies, often composed of myelin figures or lysosomes, have also been seen in a number of studies detailing the ultrastructural changes from various ototoxic insults. ${ }^{16}$ For example, cultures of human organ of Corti were used in one study to examine ultrastructural changes due to neomycin. This study demonstrated the presence of myelin bodies and vacuoles filled with lipofuscin, much like those that are described in our chick hair cells. ${ }^{9}$ Many other studies, both in mammals and birds, have shown ultrastructural changes that we have described in the chick, such as dilated mitochondria, condensed nuclear chromatin, and cytoplasmic vacuolization. ${ }^{5-7,9,12,14,17}$

From the numerous observations that we have made in this study, it appears that the process of chick hair cell death shares many properties with hair cell death in the 
mammalian organ of Corti and the mammalian vestibular labyrinth. While the anatomy of the chick inner ear may be strikingly different from that of mammals, the ultrastructural anatomy and the changes seen within degenerating hair cells are very comparable. Therefore, the observations that we have made in this study in avian hair cell degeneration are likely to shed some light on the process of hair cell death in mammals as well.

\section{INTRACELLULAR FREE CALCIUM LEVELS AFTER GENTAMICIN TREATMENT IN VITRO}

The in vivo experiments that we have performed have revealed a pattern of anatomical changes in hair cell morphology and ultrastructural abnormalities that develop after acute gentamicin toxicity that is very similar to that seen in mammals. This similarity suggests to us that the cellular mechanisms of hair cell death in mammals and in birds also share some similarities. In order to investigate some of the metabolic changes in hair cells after aminoglycoside exposure, we have implemented in vitro methods that have been previously described. For these experiments, we have used a culture preparation of the mature avian sensory epithelium from the basilar papilla that was developed primarily to study hair cell regeneration. ${ }^{3}$ The explanted epithelium provides a flat, single layer of hair cells and supporting cells that is ideal for performing in vitro experiments that require imaging techniques (FIG. 8). These tissues were ideal for investigating acute metabolic changes in hair cells after exposure to gentamicin.

For these experiments, chicks 5-7 days old were killed, the temporal bones were rapidly dissected under sterile conditions, and the basilar papilla were isolated in media. The tegmentum vasculosum, the avian analog of the stria vascularis, was removed under sterile conditions, and the tectorial membrane was then removed after digestion with collagenase. The sensory epithelium layer was elevated off of the basement membrane and was plated and grown in culture for $48 \mathrm{~h}$. These sensory epithelium explants have been successfully maintained in culture for up to 11 days, and therefore, provide a much more stable substrate than isolated single hair cells on which to perform short-term experiments. The effect of gentamicin on these cultured hair cells is also readily apparent. Tissue cultures grown in the presence of gentamicin grow supporting cells only, and all of the hair cells are effectively eliminated by the use of gentamicin (FIG. $8 \mathrm{~B}$ and $8 \mathrm{C}$ ). ${ }^{3}$

The first goal was to examine changes in intracellular free calcium in hair cells during exposure to gentamicin. Calcium is well-recognized as a critical second messenger in normal cell function, and changes in intracellular free calcium have been extensively studied in the process of cell death. Regulatory dysfunction of the tight control of calcium levels can result in a cell's demise. Prolonged periods of elevated intracellular calcium will initiate programmed cell death by activating endonucleases, phosphatases, and proteases that are dependent on calcium. ${ }^{18}$ In addition, an excess of intranuclear calcium can activate gene expression and initiate cell cycle progression, and in some cases, cell death. ${ }^{19}$

In the hair cell, changes in intracellular calcium have been studied both in the context of normal homeostasis and in damaged hair cells. In the case of hair cell damage 

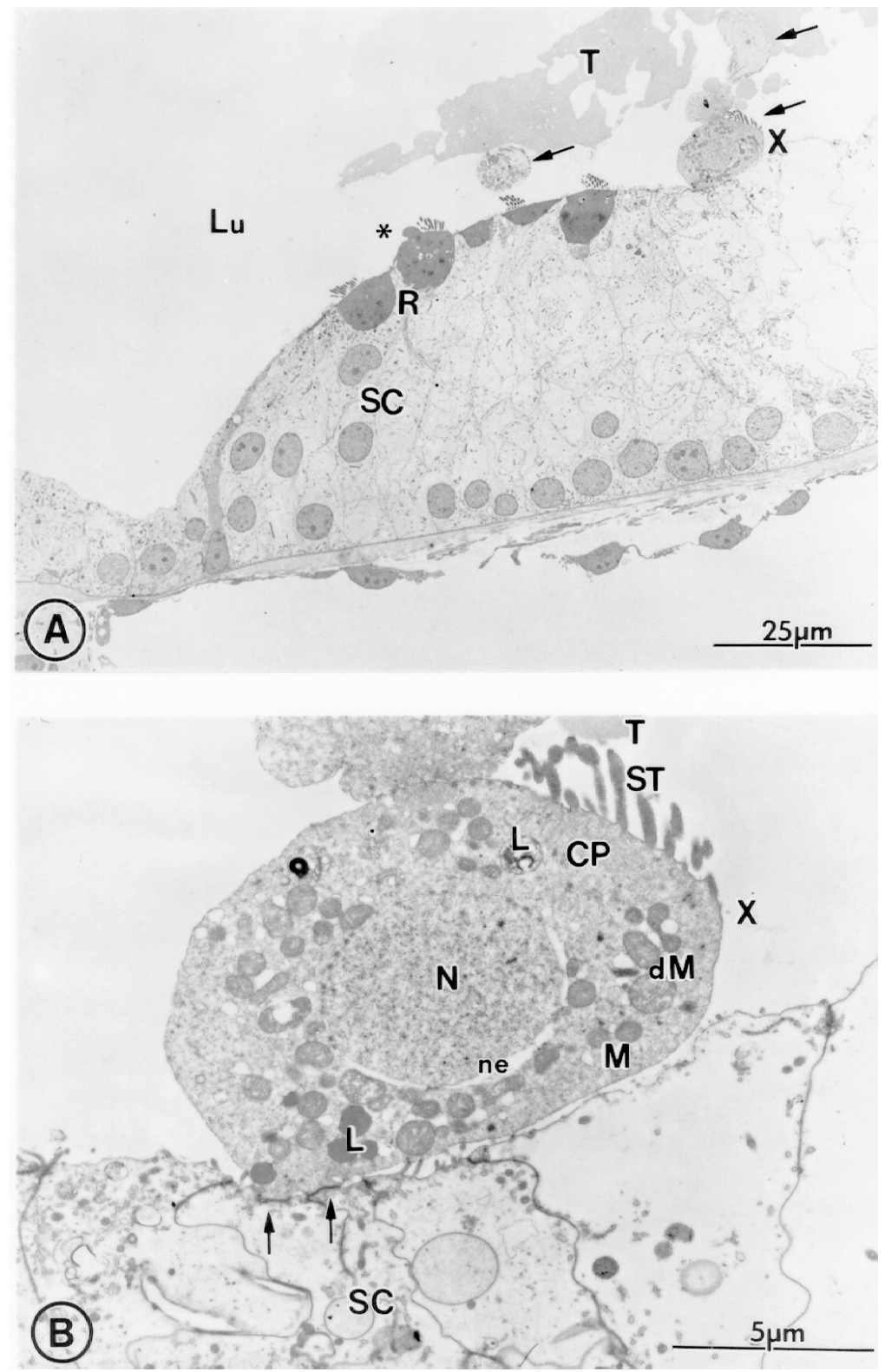

FIGURE 6. (A) Basilar papilla $24 \mathrm{~h}$ postinjection. Few rounded hair cells (R) remain and some of these show blebbing (asterisk) into the lumen (Lu). Other extruded hair cells (arrows) are enmeshed in the tectorial membrane (T). The cell at the $\mathrm{X}$ is enlarged below. (B) Higher magnification, hair cell $24 \mathrm{~h}$ postinjection. This extruded hair cell is swollen, but has an intact surface membrane and undamaged stereocilia

(Caption continues on p. 396.) 
secondary to aminoglycosides, two studies in particular have investigated changes in intracellular calcium after gentamicin exposure. Dulon et al. describe changes in calcium currents in hair cells after gentamicin treatment. ${ }^{20}$ Isolated outer hair cells from guinea pig cochleae were loaded with a fluorescent calcium dye, fluo-3. An increase in intracellular calcium was noted with fluo-3 after depolarization with potassium chloride. However, when the isolated hair cells were incubated with gentamicin, the depolarization-induced increase in intracellular calcium was prevented. This change in response to potassium chloride was attributed to an inhibition of calcium influx, although this was not directly demonstrated. Kroese et al. examined voltage clamp recordings from acutely isolated bullfrog sacculus hair cells and showed blockade of transduction channels after ionophoretic application of gentamicin. ${ }^{21}$ Based on these two studies, we expected that intracellular calcium concentration would decrease in hair cells exposed to gentamicin. However, what we discovered was quite different.

Explants of chick sensory epithelium were grown in culture for $48 \mathrm{~h}$ and then loaded with Fura 2/AM, an indicator of intracellular free calcium. The cells were then mounted on an inverted epifluorescence microscope, and intracellular free calcium levels were recorded during continuous perfusion of culture media containing gentamicin. Images were recorded every $30 \mathrm{~s}$ for a total of $60 \mathrm{~min}$.

Thirty micromolar gentamicin failed to cause any significant change in the intracellular calcium concentration (FIG. 9). The levels of intracellular calcium were stable throughout the entire experiment and were identical to the calcium levels in the control explants. However, at higher doses of $0.3,3$, and $30 \mathrm{mM}$ gentamicin, there was a dose-dependent increase in the concentration of intracellular calcium by 10-15 minutes after exposure to gentamicin (FIG 9).

Intracellular calcium levels can be influenced by a number of factors including direct influx through various kinds of calcium channels, release of calcium by mitochondria and endoplasmic reticulum, binding of calcium by cytoplasmic proteins, and extrusion of calcium by specific membrane-associated calcium pumps. Low voltage-gated calcium channels (L-type) are membrane-bound proteins that can be selectively inhibited by a class of drugs, the dihydropyridines. Nifedipine, one of the dihydropyridines, was found to inhibit the increase in intracellular calcium induced by gentamicin (FIG. 10). This result suggests that one source of elevated calcium is an influx of extracellular calcium through L-type calcium channels. The L-type voltageoperated calcium channel has been identified in the chick hair cell and appears to reside on the inferolateral surface of the cell. ${ }^{22}$ How aminoglycosides could modulate this channel has not been adequately assessed at the present time.

(FIG. 6, continued.) (ST) attached to the tectorial membrane (T), and an intact cuticular plate (CP). There are several degenerative elements in the cell. The nucleus (N) has a dispersed and homogeneous chromatin and an enlarged nuclear envelope (ne). Several inclusion bodies occur (L) and the usual ribosomal arrays are generally lacking. Several swollen mitochondria (dM) are seen as well as normal ones (M). Arrows indicate persisting intercellular junctions between the extruded hair cell and underlying supporting cells processes (SC). 

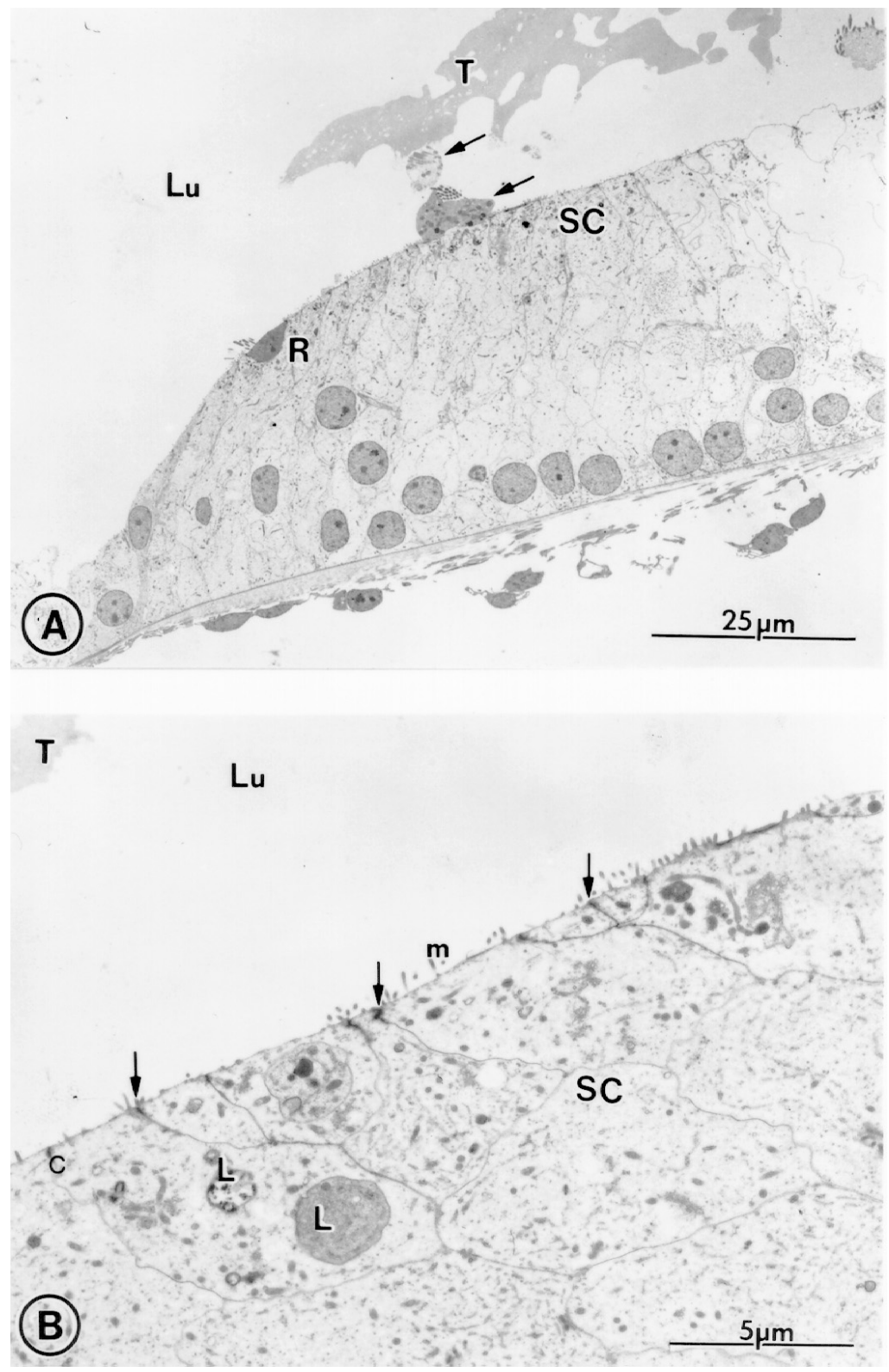

FIGURE 7. (A) Basilar papilla, $24 \mathrm{~h}$ postinjection. There are rare remaining hair cells that are rounded $(\mathrm{R})$ and several extruded hair cells (arrows). A large expanse of supporting cell processes (SC) makes up the lumenal surface. (B) Higher magnification, apical surface $24 \mathrm{~h}$ postinjection. The reticular lamina, devoid of hair cells, is entirely made up of expanded supporting cell processes (SC) that have the usual intercellular junctions (arrows) at the lumenal surface $(\mathrm{Lu})$. The supporting cells also contain a few inclusions bodies $(\mathrm{L})$. Tectorial membrane $(\mathrm{T})$ is present, but no longer in contact with hair cells. 

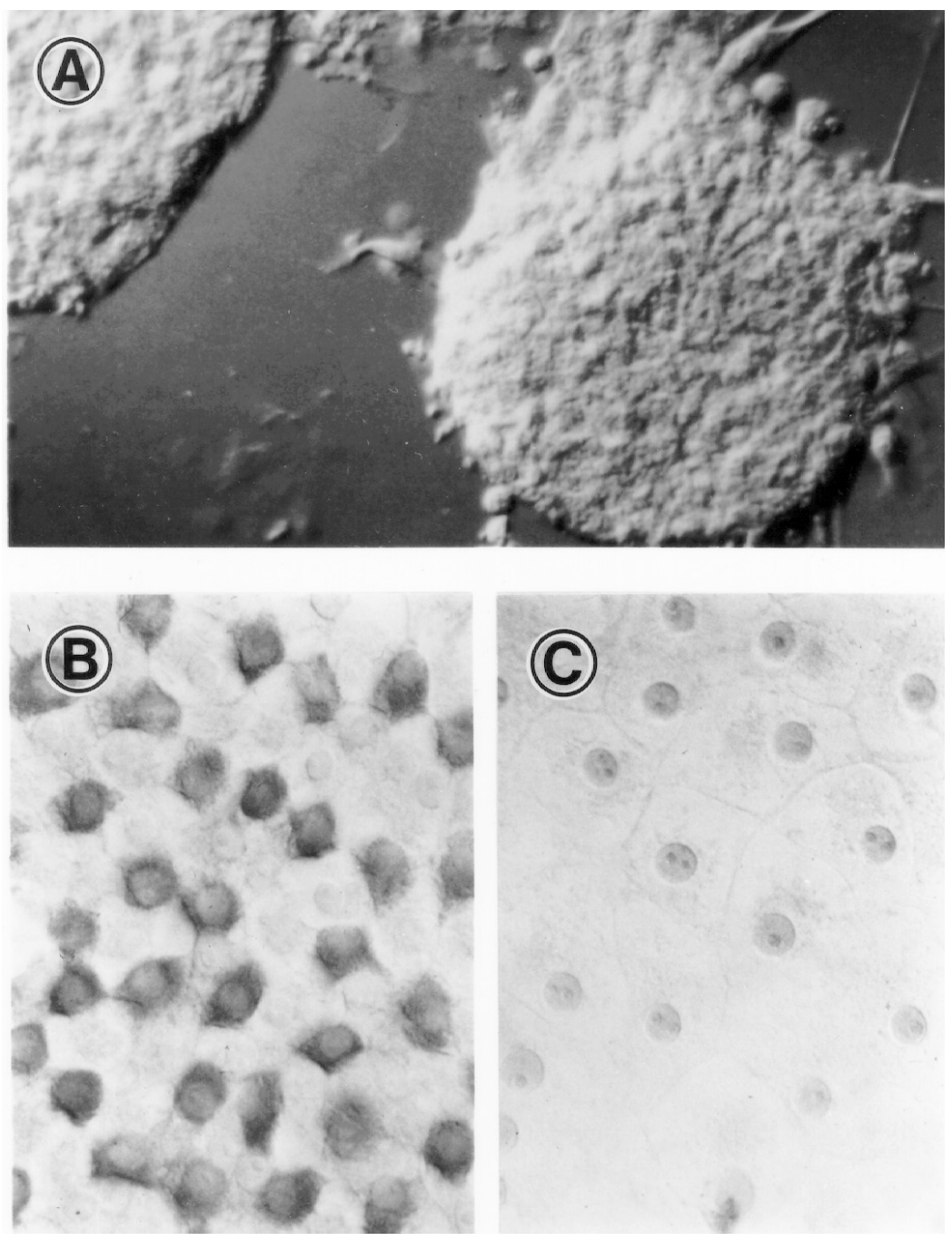

FIGURE 8. (A) Phase contrast light microscopic image of sensory epithelium explant from mature chick basilar papilla, grown in culture for $48 \mathrm{~h}$. (B) Sensory epithelium explant stained for calmodulin, a specific marker for hair cells. Note multiple cells, stained positive for calmodulin. (C) Sensory epithelium explant stained for calmodulin after $24 \mathrm{~h}$ incubation in gentamicin $3 \mathrm{mM}$. Note complete absence of hair cells. (From Stone et al. ${ }^{3}$ Reprinted with permission.) 


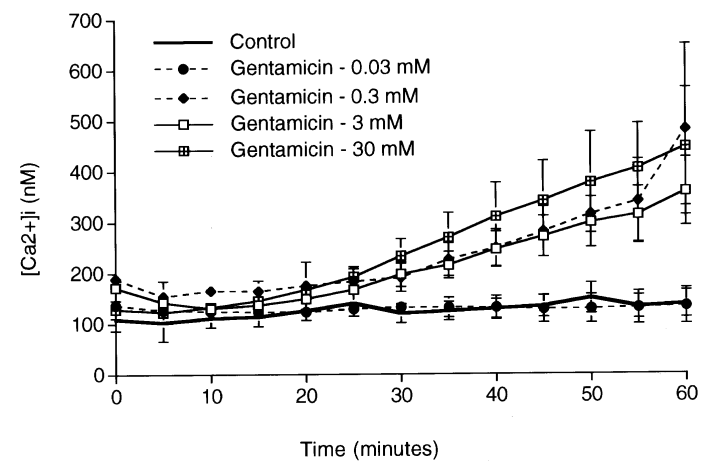

FIGURE 9. Intracellular free calcium levels after exposure to gentamicin in vitro. Sensory epithelium explants were exposed to gentamicin at four different concentrations: $0.03 \mathrm{mM}$ (filled circle), $0.3 \mathrm{mM}$ (filled diamond), $3 \mathrm{mM}$ (empty square), 30 $\mathrm{mM}$ (crossed square), and control (solid line). Note the increase in intracellular calcium concentration with increasing concentration of gentamicin. Error bars: SEM. Control was found to be significantly different from $0.3,3$, and $30 \mathrm{mM}$, but not significantly different from $0.03 \mathrm{mM}$.

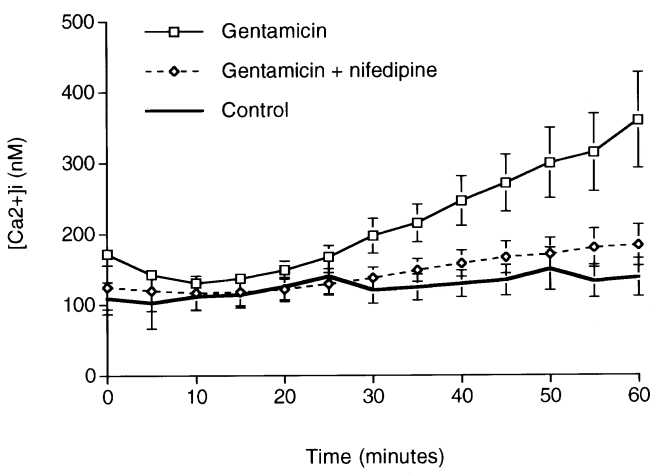

FIGURE 10. Intracellular free calcium levels after exposure to gentamicin with nifedipine in vitro. Cells were exposed to gentamicin, $3 \mathrm{mM}$ (open square), gentamicin $3 \mathrm{mM}+$ nifedipine $500 \mathrm{mM}$ (open diamond), and control (solid line). When cultured sensory epithelium cells are exposed to gentamicin in the presence of nifedipine, the previously demonstrated increase in intracellular calcium is blocked. 


\section{REACTIVE OXYGEN SPECIES AFTER GENTAMICIN TREATMENT IN VITRO}

The role of reactive oxygen species in the process of cell death has been invoked and extensively investigated in cases of cell death in the central nervous system. The importance of free radicals in the process of ototoxic hair cell death has been implicated by numerous studies that demonstrate the protective effect of antioxidant therapy against the adverse effects of known ototoxic agents. ${ }^{23-27}$ However, previous studies have not actually demonstrated an increased production of reactive oxygen species in hair cells after exposure to aminoglycosides. We developed a new technique using fluorescent vital dyes to detect the presence of reactive oxygen species after acute drug exposure. ${ }^{28} \mathrm{~A}$ technique very similar to the technique of calcium fluorescent imaging was employed using a dye called dihydrodichlorofluoroscin $(\mathrm{DCFH})$, which is a fluorescent marker for reactive oxygen species. In particular, DCFH reports the presence of oxygen free radicals such as peroxide and superoxide, but has been reported to detect nitrogen free radicals as well. ${ }^{29}$ After loading the cultures with DCFH for $15 \mathrm{~min}$, the cultured chick sensory epithelium cells were exposed to various concentrations of gentamicin and serially imaged over time. Unlike Fura-2/AM, DCFH is not a ratiometric dye, and therefore it does not provide absolute levels of free-radical activity. It does, however, provide information about relative changes in free-radical activity over time.

The methods used in this experiment were designed to parallel the methods of the previously described calcium study. The same tissue culture technique was used in both studies. The sensory epithelium cells were grown in culture for $48 \mathrm{~h}$, loaded with DCFH, and then imaged on the inverted microscope while being exposed to various concentrations of gentamicin. Images were taken every $10 \mathrm{~s}$ for a total of 10 min. FIgURE 11 shows four representative sensory epithelium cultures (in rows) that are followed over time (in columns). These four explants represent one control (11A), two experimental explants treated with gentamicin, 500 (11B) and 1000 (11C) $\mu \mathrm{M}$, and an explant treated with $500 \mu \mathrm{M}$ gentamicin and glutathione, $10 \mathrm{mM}$ (11D). These explants were imaged at 10,50, and $150 \mathrm{~s}$ after drug exposure, represented by the three columns. Two-dimensional data were transformed into three-dimensional images by projecting the level of fluorescent intensity onto the $z$-axis of the image. Each peak represents an individual hair cell with a level of free radical activity depicted by the brightness. The control explant (FIG. 11A) demonstrates a modest increase in fluorescent intensity over time. Explants exposed to 500 and $1000 \mu \mathrm{M}$ gentamicin (Figs. 11B and 11C) show a significant increase in fluorescent intensity over time. As expected, the explant treated with gentamicin and the antioxidant, glutathione (FIG. 11D), shows a marked diminution of the baseline fluorescent intensity as compared to the explants exposed to gentamicin alone and as compared to the control explant.

Average fluorescent intensity values for approximately 20 explants at each time point were obtained (FIG.12). An abrupt rise in the level of reactive oxygen species was consistently seen after exposure to 500 and $1000 \mu \mathrm{M}$ gentamicin. The effect occurred within the first $30 \mathrm{~s}$ of drug exposure, and the response plateaued by $120 \mathrm{~s}$. A dose-dependent rise in the production of reactive oxygen species was also demonstrated. 

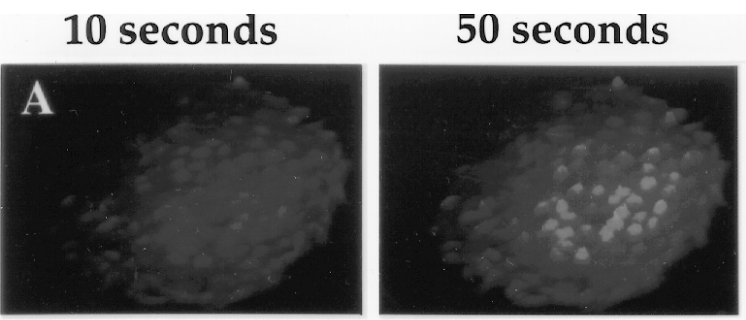

\section{0 seconds}
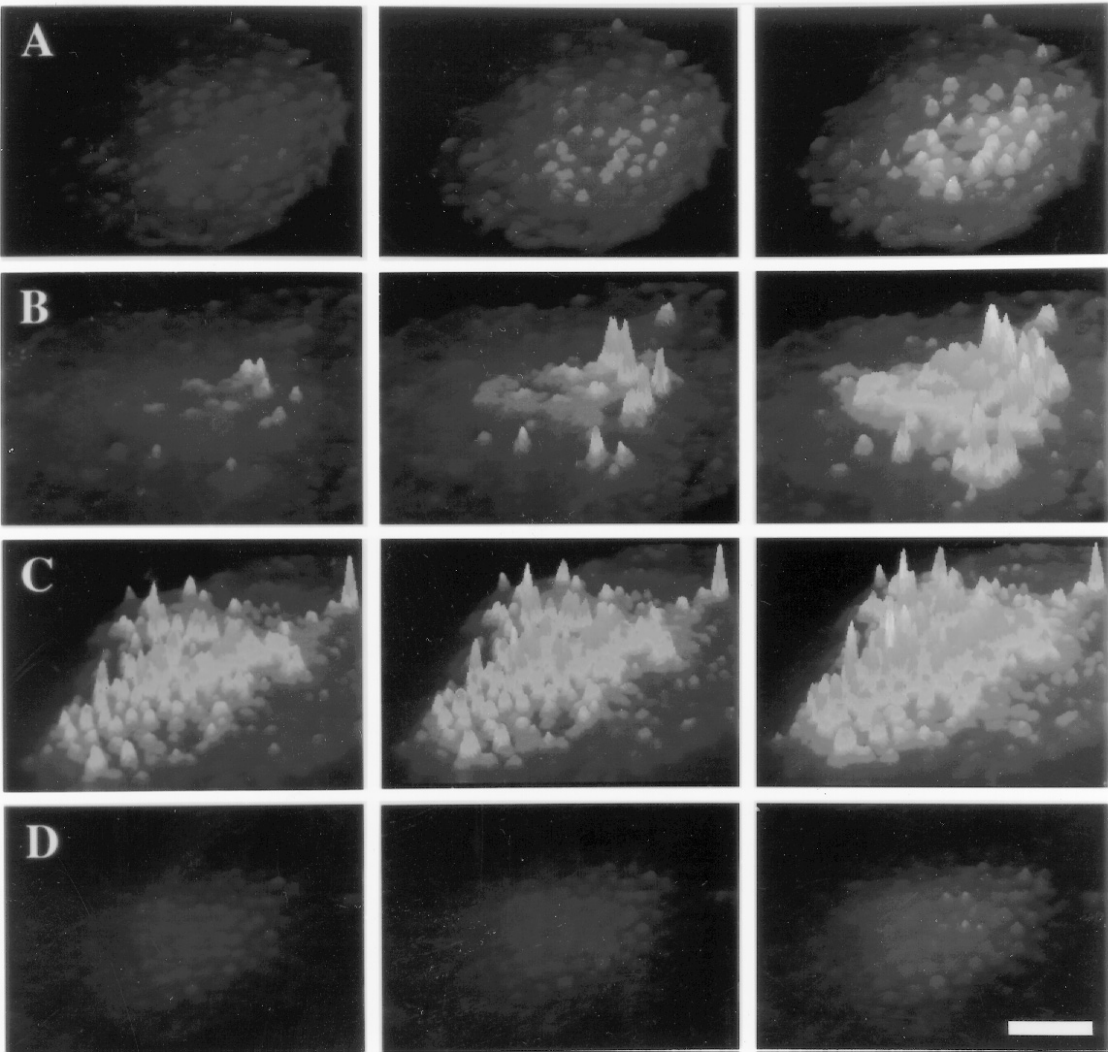

FIGURE 11. Three-dimensional representations of four live sensory epithelium explants at three time points after drug exposure. Using Image-1 computer software, the numerical fluorescent intensity is converted to a gray scale, and the two-dimensional explant is transformed into a three-dimensional image where fluorescent intensity is represented on the $z$-axis. The height of each peak represents the level of fluorescent intensity of one hair cell. (A) Control explant imaged at 10, 50, and $150 \mathrm{~s}$ after gentamicin exposure. (B) Explant exposed to $500 \mu \mathrm{M}$ gentamicin at the same time points. (C) Explant exposed to $1000 \mu \mathrm{M}$ gentamicin. (D) Explant exposed to $500 \mu \mathrm{M}$ gentamicin and $10 \mathrm{mM}$ glutathione. Here the suppression of free-radical production with the use of antioxidant is demonstrated. Scale bar: $60 \mu \mathrm{m}$. (From Hirose et al. ${ }^{31}$ Reprinted with permission.) 


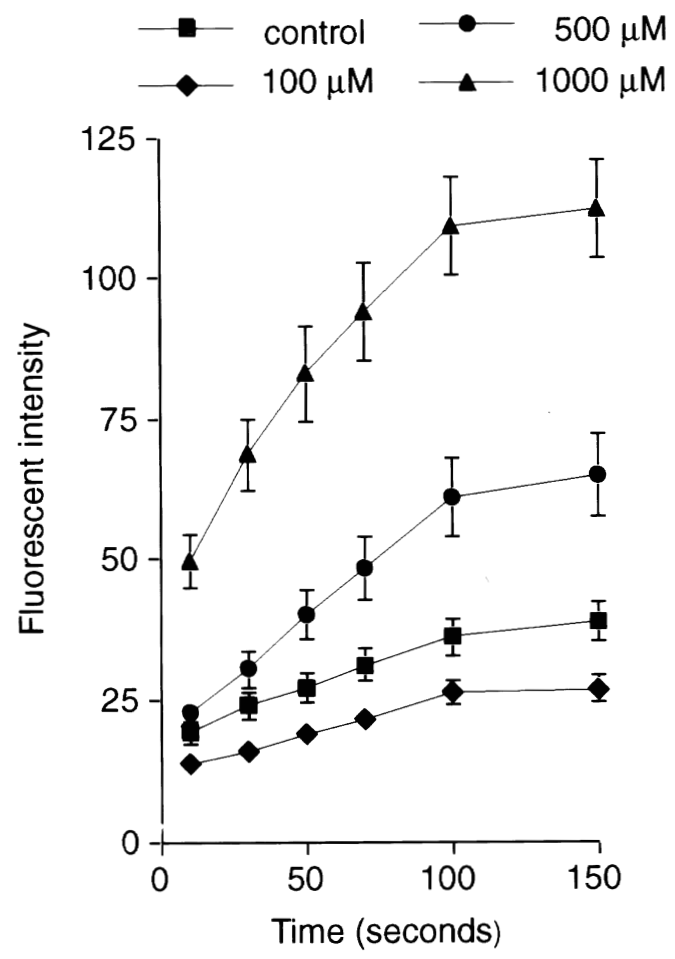

FIGURE 12. Free-radical production in hair cells after exposure to three concentrations of gentamicin. Sensory epithelium explants were imaged after addition of 100 $\mu \mathrm{M}$ gentamicin (solid diamond), $500 \mu \mathrm{M}$ gentamicin (solid circle), and $1000 \mu \mathrm{M}$ (solid triangle) gentamicin. Note the increase in free-radical production with time and with dose of gentamicin. Control, 500, and $1000 \mu \mathrm{M}$ gentamicin were found to be signficantly different, control and $100 \mu \mathrm{M}$ gentamicin were not signficantly different. Error bars: SEM. Where no error bars are shown, the SEM was smaller than the size of the symbol. (From Hirose et al. ${ }^{28}$ Reprinted with permission.)

To determine if antioxidants could inhibit free-radical production induced by gentamicin, two different antioxidants, catalase and glutathione, were applied to cultures during gentamicin exposure. These two different antioxidants were selected based on their different sizes and cell permeabilities. Catalase is a large, charged protein that is not cell-permeable, whereas glutathione is a small tri-peptide and is relatively cellpermeable. Both antioxidants were equally effective in blocking the production of reactive oxygen species by gentamicin (FIG. 13). We suspect that the formation of free radicals that we observed with gentamicin occurs either outside the cell or very superficially at the plasma membrane because entry of catalase into the cell is not required to prevent the rise in free-radical formation. 


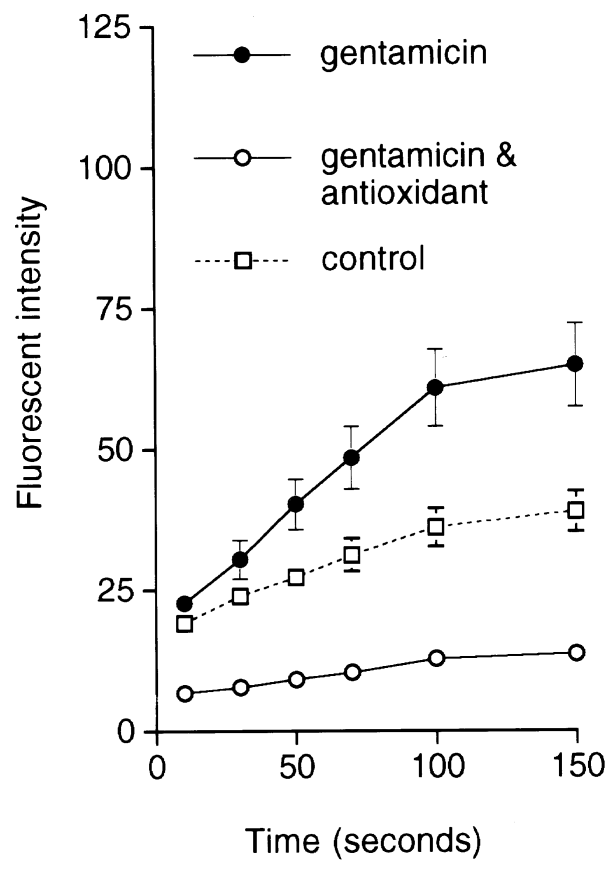

FIGURE 13. Free-radical production after exposure to antioxidant in conjunction with gentamicin. The explants treated with gentamicin alone (solid circle) demonstrated a higher level of fluorescent intensity than those treated with gentamicin and antioxidant (open circle). Also the controls (open square) showed a higher level of fluorescent intensity than the explants treated with antioxidant and gentamicin (open circle). Antioxidants effectively suppressed the increase in free radicals produced by $500 \mu \mathrm{m}$ gentamicin. Antioxidants also effectly suppressed free radicals produced in the control cultures (data not shown). Error bars: SEM. Where no error bars are shown, the SEM was smaller than the size of the symbol. (From Hirose et al. ${ }^{28}$ Reprinted with permission.)

\section{SUMMARY}

In conclusion, we have used three different techniques to investigate the dynamic changes that take place in hair cells during exposure to gentamicin. The single highdose gentamicin protocol has been implemented in vivo to look at the early ultrastructural changes that occur as early as $15 \mathrm{~h}$ after drug administration. These changes are characterized by formation of lysosomal bodies, dispersion of the polyribosomes, swelling of the mitochondria, and early blebbing and protrusion of the hair cell cytoplasm into the lumen. Within $24 \mathrm{~h}$ after drug exposure, the injury to the basilar papilla is nearly complete, with total loss of hair cells of the basal region. A number of these changes, and in particular, the loss of hair cells by the process of extrusion, appear to be a mechanism that is relatively common across species in the 
process of hair cell degeneration. The mechanism of cellular extrusion will require further study to provide a better understanding of hair cell death.

In addition, we have used tissue cultures of mature chick sensory epithelium to provide a relatively stable population of viable cells for live cell imaging. We have used this technique to demonstrate the delayed rise in intracellular calcium that is dependent on calcium influx, likely through the L-type voltage-operated calcium channel. This increase in intracellular free calcium can be effectively inhibited with the use of nifedipine, an inhibitor of the L-type calcium channel. Finally, we demonstrate the production of reactive oxygen species in hair cells after aminoglycoside exposure using this in vitro technique. The drug-induced rise in reactive oxygen species can effectively be suppressed by two different antioxidants, glutathione and catalase. Further investigation is needed to determine if these changes are necessary or sufficient to result in hair cell death. Although there is currently no apparent link between the increase in intracellular calcium and the production of free-radical species, it is possible that they are causally related. Further studies investigating second messenger pathways may link the increase in calcium to other membrane permeability changes, possibly mediated by the presence of reactive oxygen species.

\section{ACKNOWLEDGMENTS}

This work was supported by NIH Grants DC00395, DC00520, and DC00018. Special thanks to Drs. Don Henderson and Richard Salvi for organizing the Ototoxicity meeting, and to Dale Cunningham for his expertise, advise, and superb technical assistance with electron microscopy.

\section{REFERENCES}

1. Janas, J. D., D. A. Cotanche \& E. W Rubel. 1995. Avian cochlear hair cell regeneration: stereological analyses of damage and recovery from a single high dose of gentamicin. Hear. Res. 92(1-2):17-29.

2. Bhave, S. A., J. S. Stone, E. W Rubel \& M. D. Coltrera. 1995. Cell cycle progression in gentamicin-damaged avian cochleas. J. Neurosci. 15 (6):4618-4628.

3. Stone, J. S., S. G. Leaño, L. P. Baker \& E. W Rubel. 1996. Hair cell differentiation in chick cochlear epithelium after aminoglycoside toxicity: in vivo and in vitro observations. J. Neurosci. 16(19): 6157-6174.

4. Bhave, S. A., E. C. Oesterle \& M. D. Coltrera. 1998. Macrophage and microglia-like cells in the avian inner ear. J. Comp. Neurol. 398:241-256.

5. Wersall, J. \& J. E. Hawkins. 1962. The vestibular sensory epithelia in the cat labyrinth and their reactions in chronic streptomycin intoxication. Acta Otolaryngol. Stockh. 54:1-18.

6. Wenngren, B. I. \& M. Anniko. 1989. Vestibular hair cell pathology in the dancer mouse mutant. Acta Otolaryngol. Stockh. 107(3-4):182-190.

7. Bagger Sjoback, D. \& J. Wersall. 1978. Gentamicin-induced mitochondrial damage in inner ear sensory cells of the lizard Calotes versicolor. Acta Otolaryngol. Stockh. 86(1-2):35-51.

8. Richardson, G. P. \& I. J. Russell. 1991. Cochlear cultures as a model system for studying aminoglycoside induced ototoxicity. Hear. Res. 53(2):293-311. 
9. Mu, M. Y., X. PAO, T. JIE \& Y. J. YU. 1996. The alteration of ultrastructure and immunoreactivity of human embryonic organ of Corti tissue culture after exposure to aminoglycoside (neomycin) ototoxicity. Acta Otolaryngol. Stockh. 116(6):799-804.

10. Duckert, L. G. \& E. W Rubel. 1990. Ultrastructural observations on regenerating hair cells in the chick basilar papilla. Hear. Res. 48(1-2):161-182.

11. Duckert, L. G. \& E. W Rubel. 1993. Morphological correlates of functional recovery in the chicken inner ear after gentamycin treatment. J. Comp. Neurol. 331(1):75-96.

12. Sjostrom, B. \& M. Anniko. 1990. Morphologically specific vestibular hair cell degeneration in the jerker mouse mutant. Eur. Arch. Otorhinolaryngol. 247(1):51-55.

13. Li, L., G. Nevill \& A. Forge. 1995. Two modes of hair cell loss from the vestibular sensory epithelia of the guinea pig inner ear. J. Comp. Neurol. 355(3):405-417.

14. Котесha, B. \& G. P. Richardson. 1994. Ototoxicity in vitro: effects of neomycin, gentamicin, dihydrostreptomycin, amikacin, spectinomycin, neamine, spermine and poly-L-lysine. Hear. Res. 73(2):173-184.

15. Quint, E., D. N. Furness \& C. M. Hackney. 1998. The effect of explantation and neomycin on hair cells and supporting cells in organotypic cultures of the adult guineapig utricle. Hear. Res. 118:157-167.

16. Hashino, E., M. Shero \& R. J. Salvi. 1997. Lysosomal targeting and accumulation of aminoglycoside antibiotics in sensory hair cells. Brain Res. 777(1-2):75-85.

17. Friedmann, I. \& E. S. Bird. 1961. The effect of ototoxic antibiotics and of penicillin on the sensory areas of the isolated fowl embryo otocyst in organ cultures: An electronmicroscope study. J. Pathol. Bacteriol. 81:81.

18. Nicotera, P., B. Zhivotovsky \& S. OrRenius. 1994. Nuclear calcium transport and the role of calcium in apoptosis. Cell Calcium 16(4):279-288.

19. Clapham, D. E. 1995. Calcium signaling. Cell 80(2):259-268.

20. Dulon, D., G. Zajic \& J. Schacht. 1991. Differential motile response of isolated inner and outer hair cells to stimulation by potassium and calcium ions. Hear. Res. 52(1):225-231.

21. Kroese, A. B., A. Das \& A. J. HudSPeth. 1989. Blockage of the transduction channels of hair cells in the bullfrog's sacculus by aminoglycoside antibiotics. Hear. Res. 37(3):203-217.

22. Martinez Dunst, C., R. L. Michaels \& P. A. Fuchs. 1997. Release sites and calcium channels in hair cells of the chick's cochlea. J. Neurosci. 17(23):9133-9144.

23. Pierson, M. G. \& A. R. Moller. 1981. Prophylaxis of kanamycin-induced ototoxicity by a radioprotectant. Hear. Res. 4(1):79-87.

24. . Hoffman, D. W., C. A. Whitworth, K. L. Jones \& L. P. Rybak. 1987. Nutritional status, glutathione levels, and ototoxicity of loop diuretics and aminoglycoside antibiotics. Hear. Res. 31(3):217-222.

25. Hoffman, D. W., C. A. Witworth, K. L. Jones King \& L. P. Rybak. 1988. Potentiation of ototoxicity by glutathione depletion. Ann. Otol. Rhinol. Laryngol. 97(1):36-41.

26. Lazenby, C. M., S. J. Lee, E. S. Harpur \& A. Gescher. 1988. Glutathione depletion in the guinea pig and its effect on the acute cochlear toxicity of ethacrynic acid. Biochem. Pharmacol. 37(19):3743-3747.

27. Gabaizadeh, R., H. Staecker, W. Liu, R. Kopke, B. Malgrange, P. P. Lefebvre \& T. R. VAN De Water. 1997. Protection of both auditory hair cells and auditory neurons from cisplatin induced damage. Acta Otolaryngol. Stockh. 117(2):232-238.

28. Hirose, K., D. M. Hockenbery \& E. W Rubel. 1997. Reactive oxygen species in chick hair cells after gentamicin exposure in vitro. Hear. Res. 104(1-2):1-14.

29. Possel, H., H. Noack, W. Augustin, G. Keilhoff \& G. Wolf. 1997. 2,7-Dihydrodichlorofluorescein diacetate as a fluorescent marker for peroxynitrite formation. FEBS Lett. 416(2): 175-178 